\title{
Novos equinoides (Echinodermata: Echinoidea) do Albiano da Bacia do Araripe, nordeste do Brasil
}

\author{
Cynthia Lara de Castro Manso ${ }^{1}$ \& Maria Helena Hessel ${ }^{2}$
}

\begin{abstract}
Resumo Neste trabalho são registradas as espécies de Echinoidea Douvillaster benguellensis (Loriol 1888) e Hemiaster proclivus Cotteau, Peron \& Gauthier 1878 nas camadas mais superiores do Membro Romualdo da Formação Santana (Albiano) da região de Rancharia, Pernambuco, porção oeste da Bacia do Araripe. Com este registro, amplia-se para áreas mais setentrionais e ocidentais a paleodistribuição geográfica destas espécies, antes encontrados das formações Riachuelo (Aptiano-Albiano) da Bacia de Sergipe-Alagoas, em Sergipe, e em Catumbela da Bacia de Benguela, em Angola. De acordo com a morfologia funcional das carapaças, na Bacia do Araripe $H$. proclivus teria vivido no interior de sedimentos arenosos de granulometria média em ambiente marinho raso, e $D$. benguellensis teria vivido semienterrado em ambiente mais profundo, onde as condições de circulação e oxigenação das águas eram mais restritas.
\end{abstract}

Palavras-chave: Echinoidea, Eocretáceo, Formação Santana.

\begin{abstract}
New echinoids (Echinodermata: Echinoidea) from Albian of Araripe Basin, northweastern Brazil. In this paper are registered the Echinoidea species Douvillaster benguellensis (Loriol 1888) and Hemiaster proclivus Cotteau, Peron \& Gauthier 1878 in the upper beds of the Romualdo Member, Santana Formation (Albian), from the Rancharia District, Pernambuco State, western portion of the Araripe Basin. With this registry, extends the geographical paleodistribution of these species, before found in the Riachuelo Formation (Aptian-Albian) and in the Sergipe-Alagoas Basin in Brazil, and Catumbela Formation from the Benguela Basin in Angola. According to the functional morphology of the shells, in the Araripe Basin, H. proclivus had lived inner of sand substrate in shallow marine environment, and $D$. benguellensis had lived almost as an endofaunal organism in deeper environment, where the circulation and oxygenation of the water conditions were more restricted.
\end{abstract}

Keywords: Echinoidea, Early Cretaceous, Santana Formation.

\begin{abstract}
INTRODUÇÃO O estudo dos equinoides na Bacia do Araripe foi iniciado por Beurlen (1966), com o registro de duas espécies de cassiduloides da Formação Santana: Pyguros tinocoi e Faujasia araripensis. Mais tarde, Brito (1981) transferiu a espécie Faujasia araripensis para o gênero Pygidiolampas. Manso \& Hessel (2007), revisando a espécie P. araripensis, transferiram-na para o gênero Bothryopneustes Fourtau 1924, da família Clypeidae Lambert 1898. Até o momento nenhuma outra espécie de Echinoidea foi acrescentada àquelas descobertas por Beurlen há cerca de 50 anos. O objetivo deste trabalho é registrar, descrever e figurar mais duas espécies de equinoides ocorrentes nos estratos albianos da Formação Santana (Membro Romualdo), tecendo considerações paleoecológicas, bioestratigráficas e paleobiogeográficas.
\end{abstract}

GEOLOGIA DA BACIA DO ARARIPE A Bacia do Araripe, localiza-se entre as latitudes $7^{\circ}$ e $8^{\circ} \mathrm{S}$ e as longitudes $38^{\circ} 30^{\prime}$ e $41^{\circ} \mathrm{W}$, numa área que compreende o sul do Estado do Ceará, o noroeste de
Pernambuco e o leste do Piauí (Arai et al. 2004, Brito 1990) (Fig.1). Destaca-se na paisagem pela feição geomorfológica de chapada, cujas altitudes atingem de 600 a $1.000 \mathrm{~m}$ (Martill et al. 2007). A chapada é constituída por unidades aptianas a cenomanianas, seccionadas por escarpas erosivas e íngremes, que recobrem, em discordância angular, unidades de sequências mais antigas ou repousam diretamente sobre o embasamento cristalino. As sequências sedimentares constituintes da bacia apresentam mergulho em torno de $5^{\circ} \mathrm{W}$ (Brito 1990).

Durante o Fanerozoico, diversos eventos tectônicos reativaram antigas estruturas do embasamento cristalino, subordinando as bacias interiores do nordeste do Brasil. Dentre elas, a Bacia do Araripe é a que apresenta evolução tectono-sedimentar mais complexa, porque é uma bacia poli-histórica, constituída pela superposição de diversas sequências estratigráficas, limitadas por discordâncias, que representam o registro sedimentar de várias bacias geneticamente distintas (Assine 1992). 
Há diferentes propostas para a sequência estratigráfica da Bacia do Araripe, sendo aqui adotada a mais recente, de Assine (2007; Fig. 2).
Dentre as unidades estratigráficas da bacia, a Formação Santana é a que apresenta maior riqueza fossilífera, sendo objeto do presente trabalho. Suas camadas

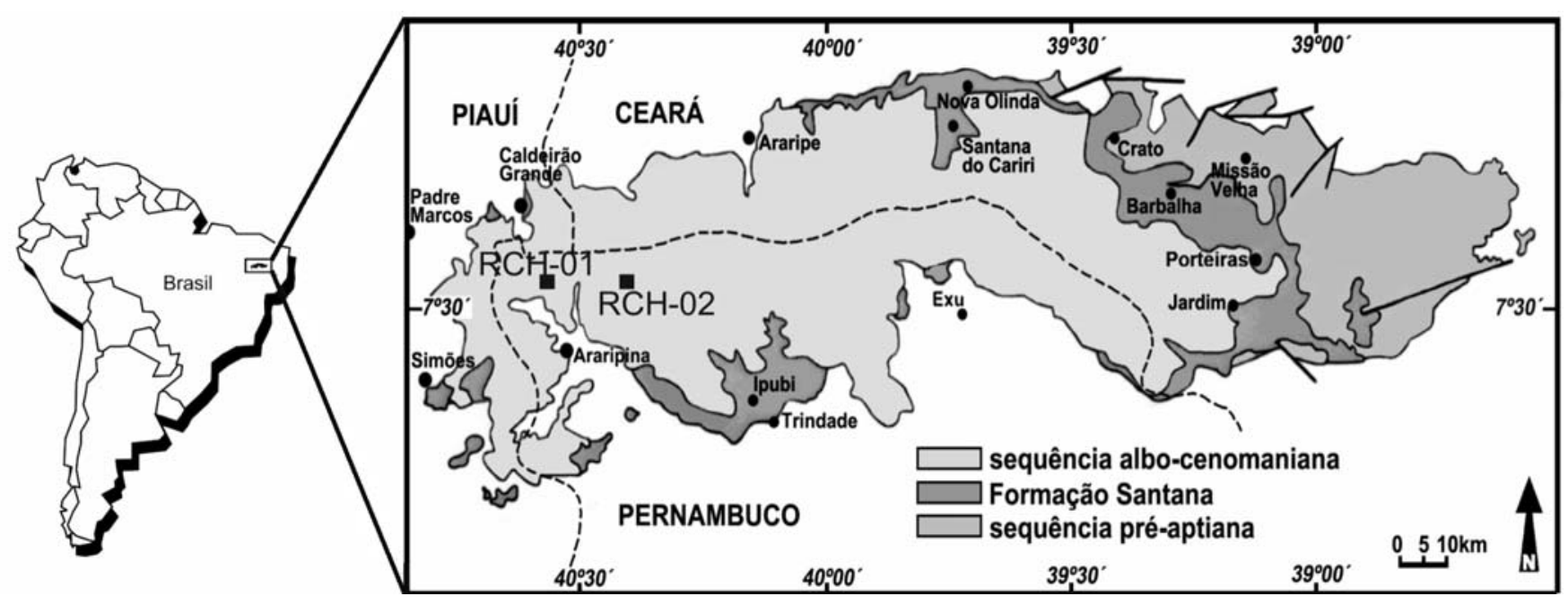

Figura 1 - Bacia do Araripe e a porção aflorante da Formação Santana onde foram encontrados os equinoides aqui descritos (modificada de Assine 2007).

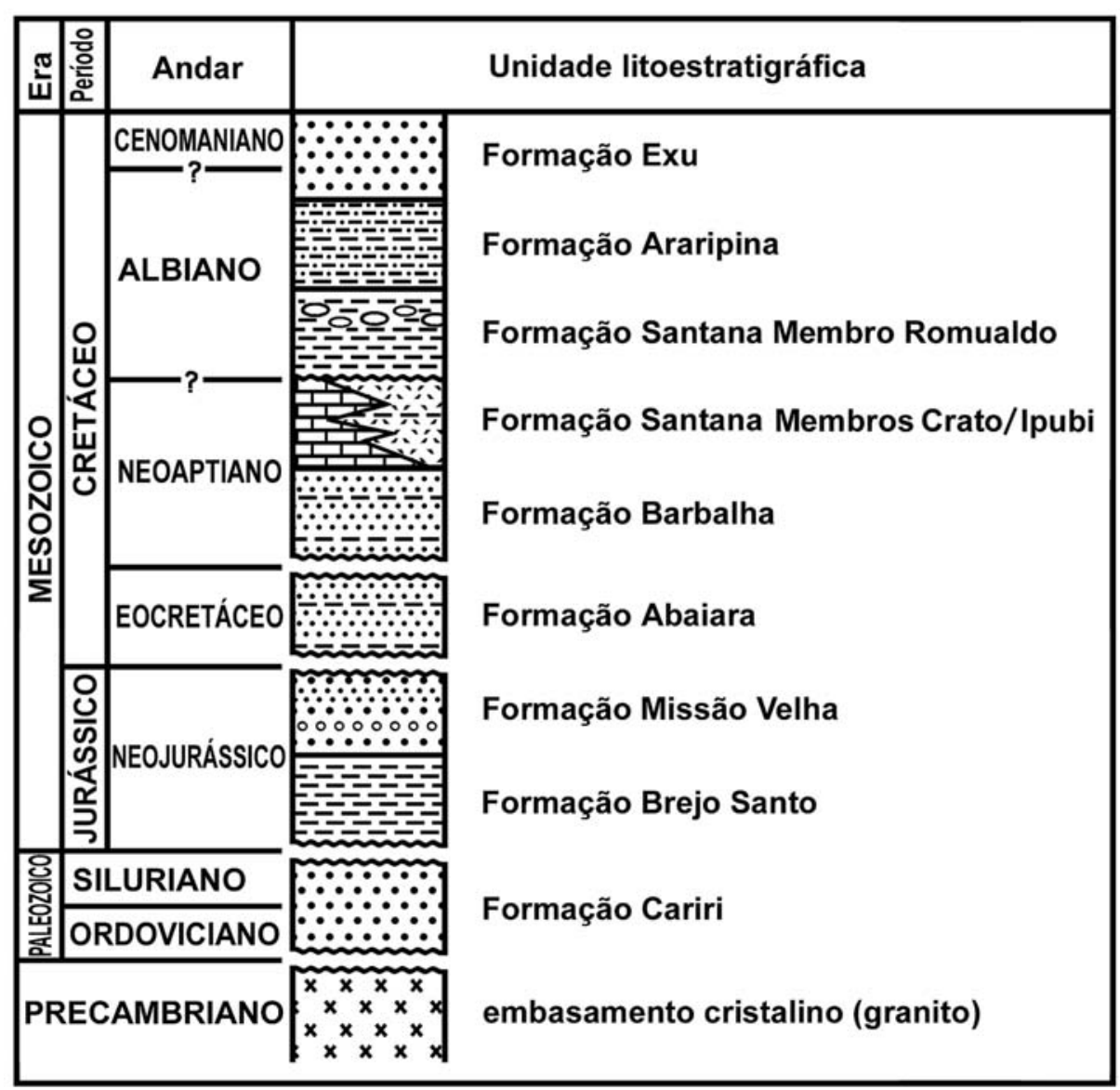

Figura 2 - Síntese da estratigrafia da Bacia do Araripe (modificada de Assine 2007). 
ocorrem no sopé das escarpas que contornam a chapada do Araripe, e no morro-testemunho Serra do Mãozinha, onde aflora sobre os estratos da Formação Barbalha, mas também diretamente sobre o embasamento na borda da bacia. Subdivide-se em três membros: o Membro Crato é constituído por calcários finamente laminados, caracterizado pela abundância e diversidade de fósseis; o Membro Ipubi, que apresenta sedimentação predominantemente evaporítica, onde se intercalam gipsita, anidrita, folhelhos e carbonatos, e que também contém fósseis; e o Membro Romualdo, superior, constituído por leitos pelítico-margosos intercalados por folhelhos betuminosos muito fossilíferos, arenitos e bancos calcários, principalmente no topo, onde ocorreu a maior influência marinha, contendo níveis de concreções carbonáticas ricas em fósseis.

O Membro Crato aflora como bancos descontínuos com espessuras que ultrapassam a $20 \mathrm{~m}$, em contato interdigitado com os evaporitos do Membro Ipubi (Heimhofer et al. 2010).

O Membro Ipubi, com espessura máxima de $30 \mathrm{~m}$, mostra uma sequência de gipsita e anidrita com folhelhos escuros intercalados. A descontinuidade das camadas deste membro, a existência de intercalações de folhelhos com conchostráceos e pirobetuminosos ricos em fragmentos vegetais carbonizados e ostracodes não marinhos, sugere que não havia uma ampla bacia evaporítica na região, e sim ambientes supramarginais sujeitos a variações do nível do lago sob um clima árido a semiárido. Embora existam diversas ocorrências em toda a bacia, as camadas de gipsita concentram-se, sobretudo, na porção oeste, de Ipubi a Araripina (Pernambuco).

O Membro Romualdo, com estimados $60 \mathrm{~m}$ de espessura, mostra em sua porção inferior arenitos finos e folhelhos interestratificados (Chagas 2006). Segundo Assine (2007), esses folhelhos adquirem coloração mais escura em direção ao topo, onde há $20 \mathrm{~m}$ de folhelhos ricos em matéria orgânica. Neles, há um nível de quase 6 m onde ocorrem concreções calcárias de diversas formas e tamanhos, muitas delas contendo restos de peixes, outros vertebrados e plantas. Poucos metros acima há arenitos finos e um nível de quase $1 \mathrm{~m}$ de espessura com concentração de restos esqueletais de moluscos marinhos e equinoides, correspondendo, segundo Assine (2007), a uma superfície de inundação máxima. A sequência sedimentar do Membro Romualdo, rica em ostracodes e palinomorfos típicos de ambientes mesohalinos (Arai \& Coimbra 1990), representa a deposição em um golfo, enseada ou sistema de lagunas costeiras, com alguma influência marinha nos tempos finais de sua deposição. Este membro parece ter sido depositado em descontinuidade erosiva de curta duração sobre o Membro Ipubi, pois na área de Nova Olinda, os arenitos e conglomerados da base do Membro Romualdo truncam as camadas de gipsita e folhelho. Na parte oeste da bacia, como, por exemplo, na mina Lagoa de Dentro (Araripina), também existem evidências de retrabalhamento, com a presença de delgado nível de paraconglomerados acima dos evaporitos (Silva 1986). Segundo esta autora, esta descontinuidade pode ser observada também em áreas mais à leste onde os evaporitos estão ausentes, sendo reconhecida por superfícies de paleocaliche e finas camadas de paraconglomerados sobrepostas ao Membro Crato.

MATERIAL E MÉTODOS O material aqui estudado provém do distrito de Rancharia, município de Araripina, Estado de Pernambuco, porção oeste da Bacia do Araripe, e foi coletado por Maria Helena Hessel em outubro de 2006. As localidades de coleta foram descritas tomando-se por base a metodologia adotada por Bengtson (1983, p. 30-31) para a bacia de Sergipe e modificada para a bacia do Araripe. Os códigos em parênteses referem-se à identificação dos afloramentos no mapa de localização. O código Ksr corresponde à Formação Santana, Membro Romualdo. Por exposição entende-se afloramento com altura inferior a $0,5 \mathrm{~m}$.

Rancharia 1(RCH-01): 7¹7'38”S e 40³1'03”W. Exposição = material solto em corte da estrada carroçável que dá acesso à Fazenda do Isaques, próximo à Rancharia

Ksr = calcário creme-claro.

Rancharia 2 (RCH-02): 7¹7'39'’S e 4021'18'’W. Exposição = material solto na superfície de uma colina na Fazenda dos Isaques, próximo à Rancharia.

Ksr = calcário creme-claro.

Os fósseis foram lavados em água corrente e preparados fisicamente com estiletes de aço. Em seguida, foram medidos, descritos sob lupa binocular e figurados. Foram efetuadas medidas do comprimento, largura e altura de todos os exemplares em melhor estado de preservação. Nos exemplares de Hemiaster proclivus foram também efetuadas medidas das pétalas anteriores, pétalas posteriores e da distância entre o sistema apical e o bordo anterior da carapaça, com o objetivo de observar as variações ontogenéticas. O material encontra-se depositado na Coleção de Invertebrados Fósseis (FPH-I) da Fundação Paleontológica Phoenix em Aracaju, Estado de Sergipe, Brasil.

\section{SISTEMÁTICA PALEONTOLÓGICA}

Spatangoida

Toxosteridae

Douvillaster benguellensis (Loriol 1888)

Figura 3 
1888 Isaster benguellensis Loriol, p. 108, est. 6-8. 1996 Douvillaster benguellensis Neumann, p. 260. 2003 Douvillaster benguellensis Manso \& Souza-Lima, p. 32-34, figuras 3 a 5.

Holótipo: não disponível, corresponde à descrição de Isaster benguellensis por Loriol (1888) p. 108.

Diagnose: carapaça ovalada a cordiforme com a superfície dorsal regularmente convexa e a ventral plana; ambulacro ímpar assentado sobre uma depressão suave; poros ambulacrais alongados em forma de fenda em todos os ambulacros; pétalas posteriores um pouco mais curtas e divergentes que as anteriores (modificada de Loriol 1888).

Material: um exemplar com restos da carapaça, depositado na Fundação Paleontológica Phoenix sob o número FPH-1537-I, coletado na localidade Rancharia 1.
Descrição: carapaça mal preservada, de contorno cordiforme, medindo $36 \mathrm{~mm}$ de comprimento por $35 \mathrm{~mm}$ de largura e 16,5 mm de altura. Tubérculos de tamanhos diferentes com plataforma crenulada na região aboral. Poros do ambulacro III (anterior) em forma de fenda curta semelhante aos poros das pétalas pares (Fig. 3C). Sulco ambulacral anterior longo, pouco profundo chegando ao âmbitus. Pétalas pares anteriores longas (aproximadamente $17 \mathrm{~mm}$ de comprimento) e abertas, em um sulco raso. Pétalas pares posteriores com aproximadamente $11 \mathrm{~mm}$ de comprimento. Disco apical e peristômio não foram preservados.

Distribuição estratigráfica e geográfica: embora o sistema apical não tenha sido observado, todas as demais feições morfológicas se assemelham àquelas observadas nos exemplares referidos a Douvillaster benguellensis (Loriol 1888) da Bacia de

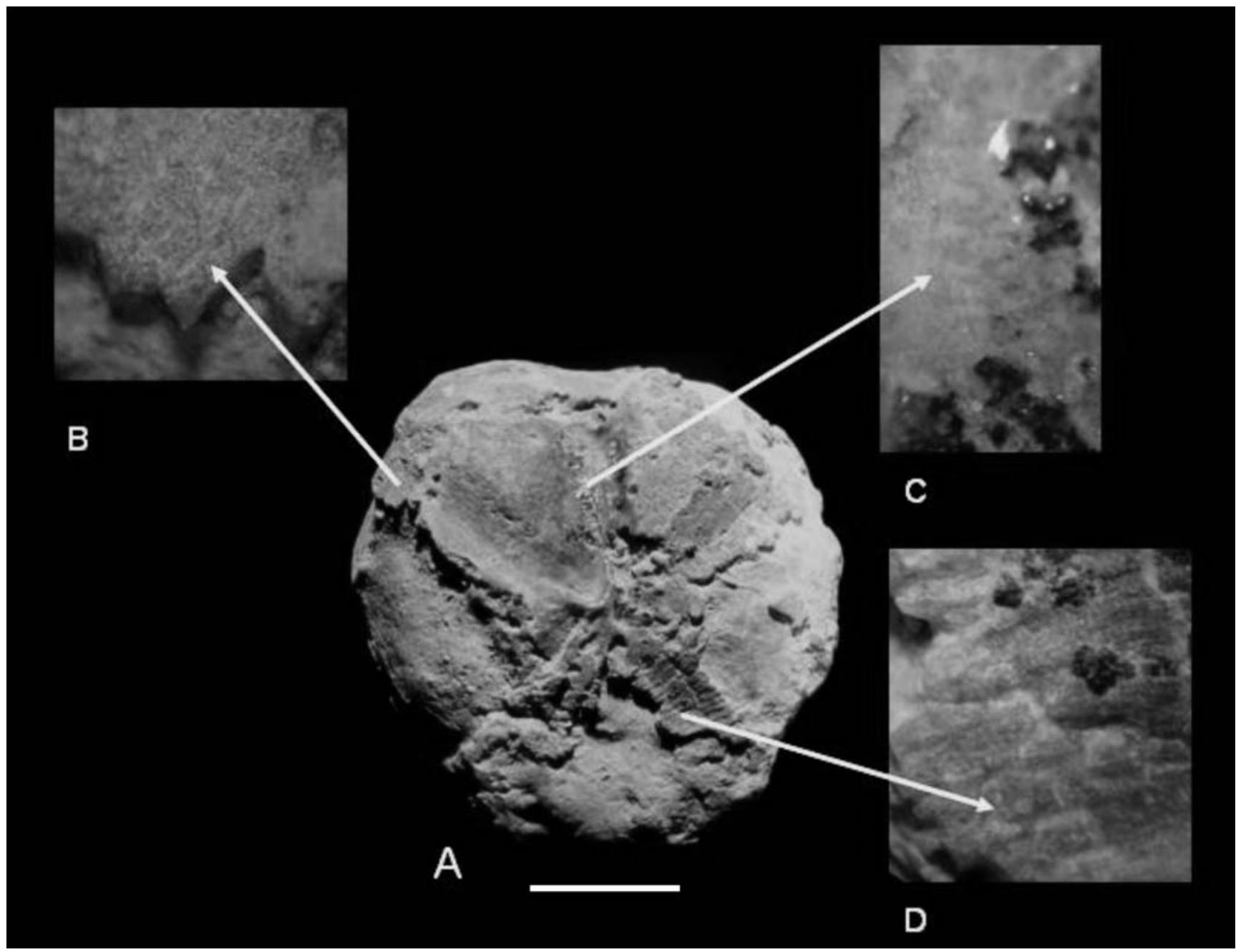

Figura 3 - Douvillaster benguellensis (Loriol) do Albiano (Membro Romualdo, Formação Santana) da Bacia do Araripe, Pernambuco: A - vista aboral; B - vista aproximada dos pares de poros da extremidade da pétala par anterior; C - vista aproximada dos pares de poros do ambulacro III em forma de fenda curta; D - vista aproximada dos pares de poros na altura da metade da pétala posterior. Barra de escala: $10 \mathrm{~mm}$. 
Sergipe-Alagoas no Estado de Sergipe por Manso \& Souza-Lima (2003). D. benguellensis foi registrada primeiramente na Formação Catumbela, Neoalbiano da Bacia de Benguela em Angola (Loriol 1888). No Brasil, esta espécie foi até o momento registrada nos calcários neoaptianos da Formação Riachuelo em Sergipe (Manso \& Souza-Lima 2003). Assim sendo, com o presente estudo, $D$. benguellensis tem sua distribuição geográfica ampliada para locais mais setentrionais, na porção oeste da Bacia do Araripe em Pernambuco, onde pode ser encontrada no Membro Romualdo da Formação Santana. As camadas onde ocorrem nesta bacia são datadas como neoalbianas (Coimbra et al. 2002), como as formas africanas.

Observações: de acordo com Smith (2011), o gênero Douvillaster é idêntico ao gênero Macraster, sendo este último utilizado para nomear as espécies americanas, enquanto Douvillaster é utilizado para nomear as espécies norte-africanas e européias. O exemplar aqui descrito encontra-se parcialmente dolomitizado.

Hemiasteridae

Hemiaster proclivus Cotteau, Peron \& Gauthier 1878 Figuras 4 e 5; tabela 1

1878 Hemiaster proclivus Peron \& Gauthier - Cotteau, Peron \& Gauthier, p. 121, est. 5:1-4.

1887 Hemiaster cristatus Stoliczka? - White, p.261 (pars). 1888 ?Epiaster catumbellensis Loriol - Loriol, p.112, est. 8:3-6.

1931 Hemiaster proclivus Gauthier - Lambert, est. 4:23. 1932 Hemiaster proclivus Gauthier - Lambert, p.114. 1937 Hemiaster jacksoni Maury - Maury, p. 279 (pars). 1959 Hemiaster cf. Hemiaster cranium Cooke - Santos \& Cunha, p.12, est. 1:5; 2:1-6.

1981 cf. Hemiaster cranium Cooke - Brito, p. 402.

1991 Hemiaster proclivus Cotteau, Peron \& GauthierSmith, est. 11D-F:43, 44, 45A-C.

2003 Hemiaster proclivus Manso, p.106, est.X:43.

Lectótipos: MN 4663-I, DGM 4542-I, DGM 4543-Ia, b, c.

Diagnose: um Hemiaster com a carapaça moderadamente deprimida; parafascíolo peripetálico largo e difuso também na região anterior da carapaça; contorno fracamente cordiforme nos indivíduos maiores; ambulacro anterior relativamente largo, com a superfície aboral não muito inclinada; pétalas posteriores relativamente curtas, com $60 \%$ do comprimento das pétalas anteriores (modificada de Smith 1991).

Material: 11 exemplares completos depositados na Fundação Paleontológica Phoenix sob os números FPH-1527-I a FPH-1536-I e FPH-1538-I coletados nas localidades Rancharia 1 e Rancharia 2.
Descrição: carapaças de contorno cordiforme, arredondadas na região anterior e levemente quadradas na região posterior. As carapaças variaram entre 15,0 a 24,5 mm de comprimento, sendo a largura aproximadamente de $94 \%$ do comprimento da carapaça (Tab. 1). Sulco anterior raso mesmo naqueles exemplares com $15 \mathrm{~mm}$ de comprimento. O ponto mais alto da carapaça está localizado na metade posterior, entre o sistema apical e o bordo posterior. Tubérculos de tamanhos diferentes estão espalhados sobre a região aboral da carapaça, sendo que os maiores estão localizados próximos ao sistema apical e lateralmente ao sulco ambulacral anterior.

O periprocto é oval e encontra-se na região posterior, localizado no alto de um leve declive, podendo assim ser visualizado da região aboral. Sete a oito pares de poros podem ser observados, situados lateralmente na carapaça e abaixo do periprocto.

O sistema apical encontra-se aproximadamente no centro da carapaça, de 50 a $54 \%$ da distância entre o sistema apical e a região anterior da carapaça. É etmofráctico (Fig. 5F), com os poros genitais formando um quadrado aproximado. As placas genitais 1 e 4 estão em contato, e a placa genital 2 (madreporita), com 18 poros, é quase do mesmo tamanho que a placa genital 3 no exemplar FPH-1532-I (que possui $18 \mathrm{~mm}$ de comprimento de carapaça). Já no exemplar FPH-1536-I, de 14 mm de comprimento, a placa genital 2 é bem maior do que a placa genital 3, mas, mesmo assim, não separa as placas genitais 1 e 4 que estão em total contato.

Ambos os pares de pétalas anteriores e posteriores são retilíneos e formados por poros alongados. Em todos os exemplares examinados, as pétalas pares anteriores são mais longas do que as pétalas pares posteriores. O exemplar FPH-1532-I possui 20 pares de poros nas pétalas pares anteriores e 13 pares nas pétalas pares posteriores, ou seja, o par posterior é $65 \%$ do tamanho do par anterior.

O ambulacro anterior é levemente sulcado e se estende do sistema apical ao bordo anterior da carapaça. Os poros ambulacrais são arredondados e iguais, sendo que cada poro do mesmo par está separado por um pequeno tubérculo. No exemplar FPH-1536-I (de $14 \mathrm{~mm}$ de comprimento) existem 12 pares de poros, e no exemplar FPH-1527-I (com carapaça de 24,5 mm de comprimento) foram contados 18 pares de poros.

Um parafascíolo peripetálico pode ser observado disperso e sem margens definidas ao redor das pétalas, inclusive formando uma banda larga na extremidade dos poros do ambulacro III no exemplar FPH-1527-I, com carapaça de 24,5 mm de comprimento. Neste parafascíolo são encontrados tubérculos primários dispersos.

O peristoma está localizado na região oral e mais próximo da região anterior. É semipentagonal 


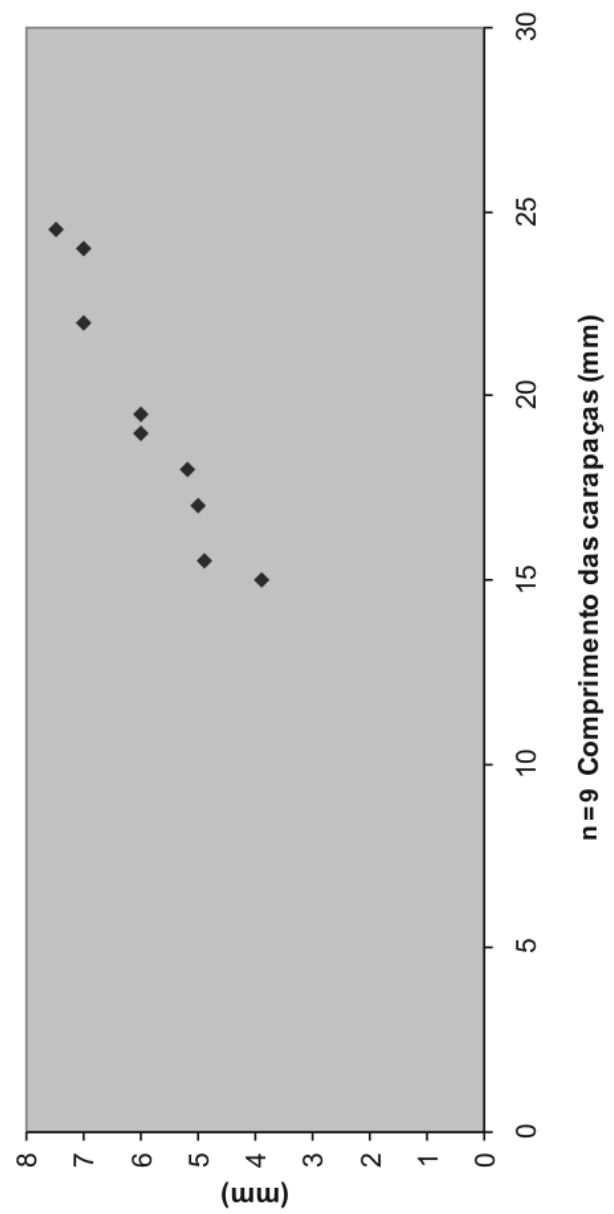

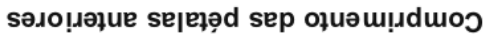

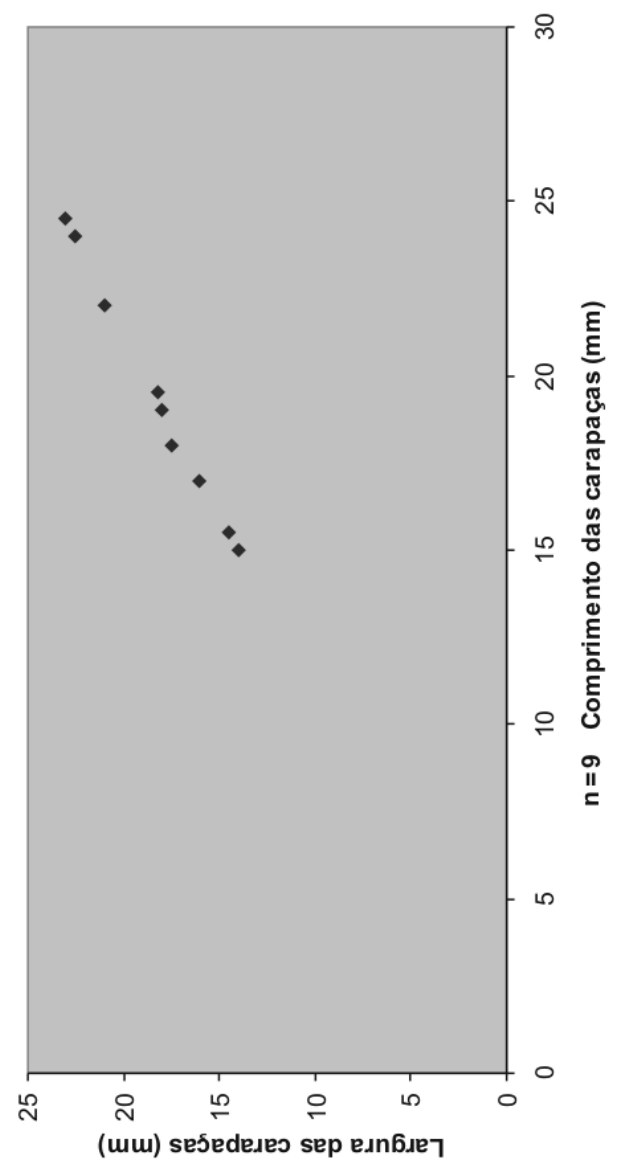

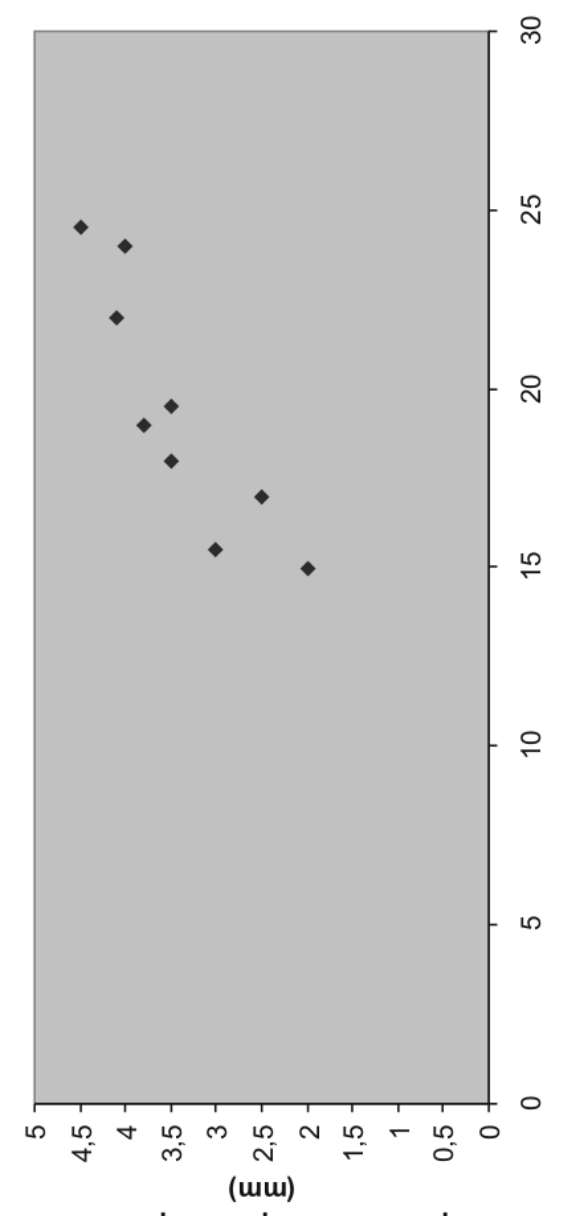

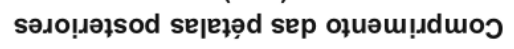

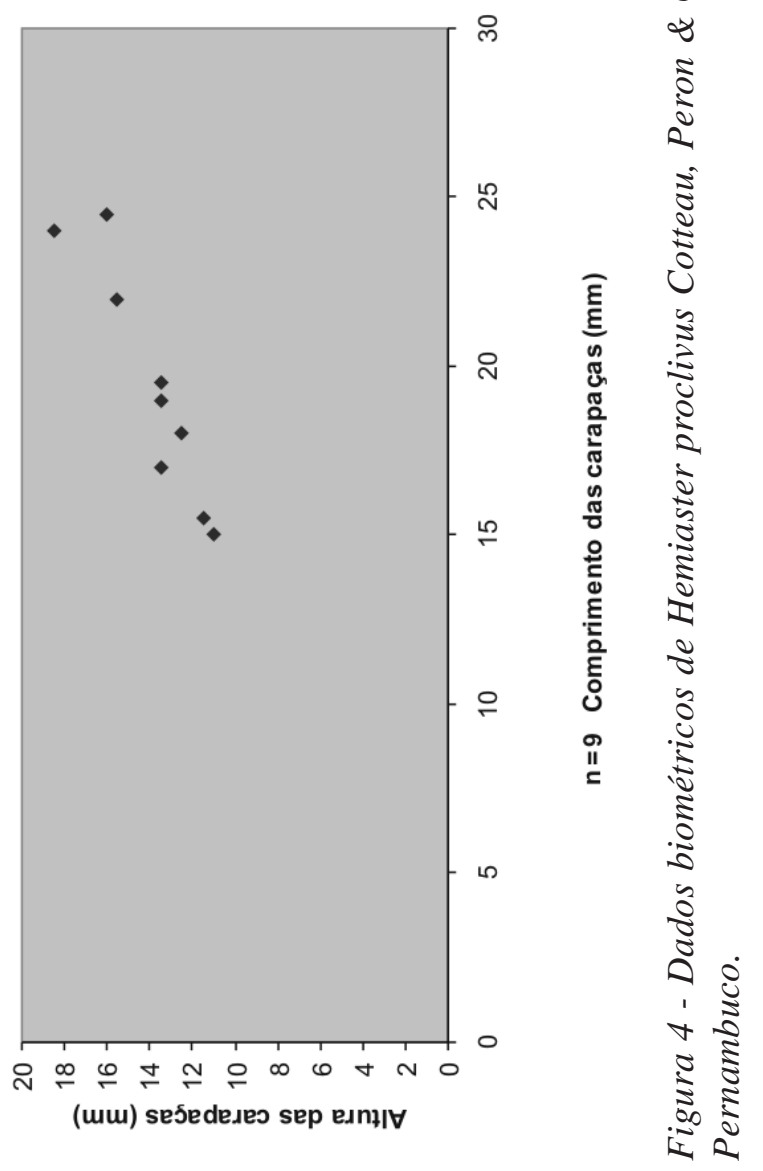




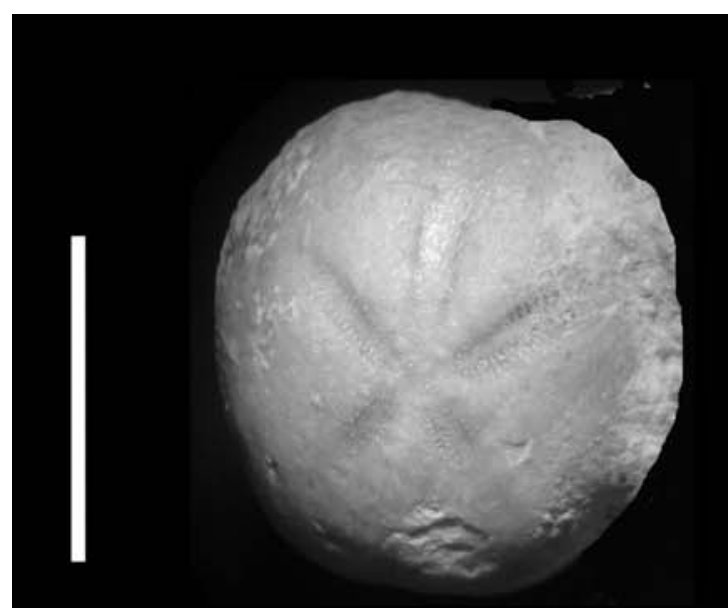

A
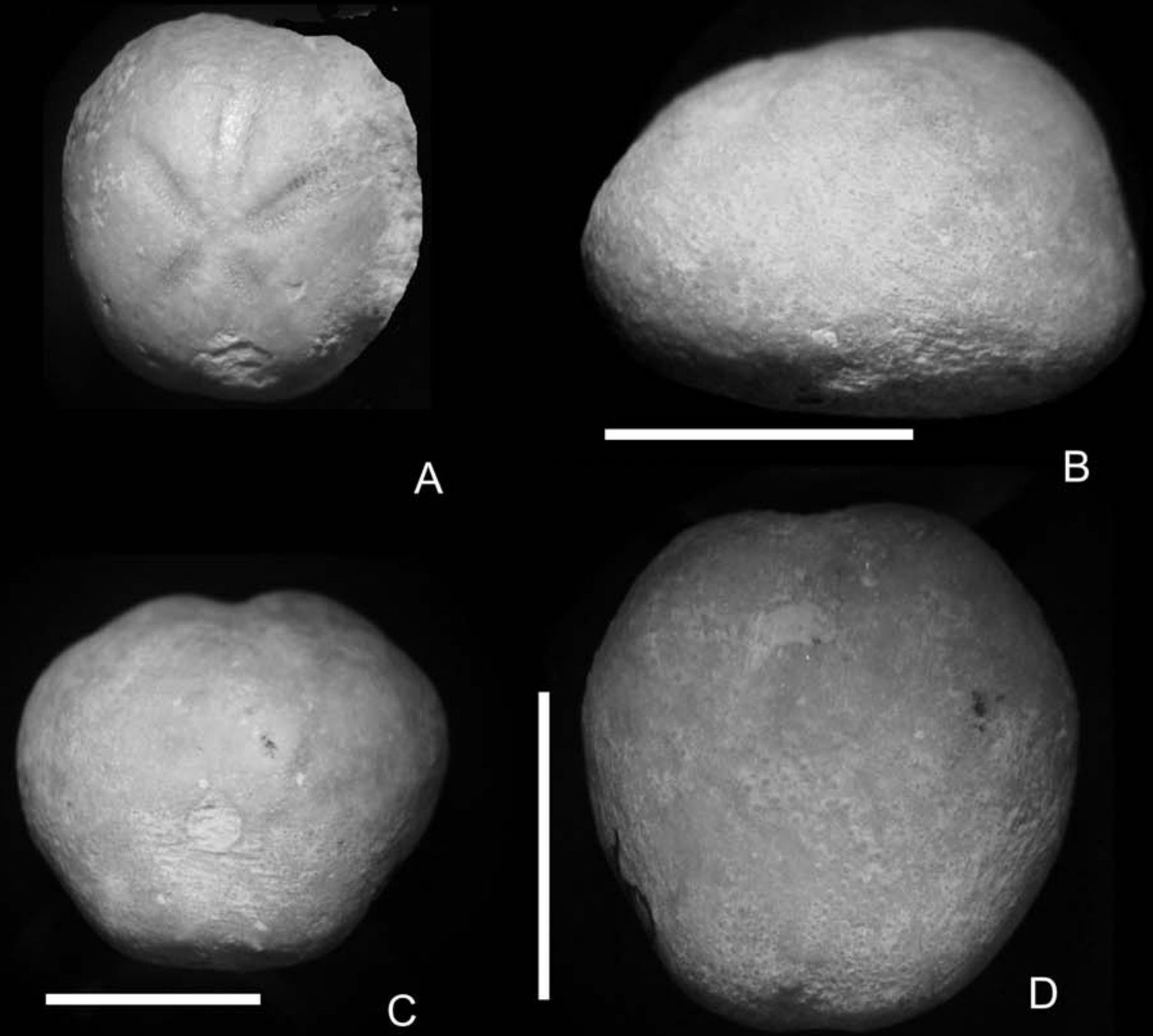

C
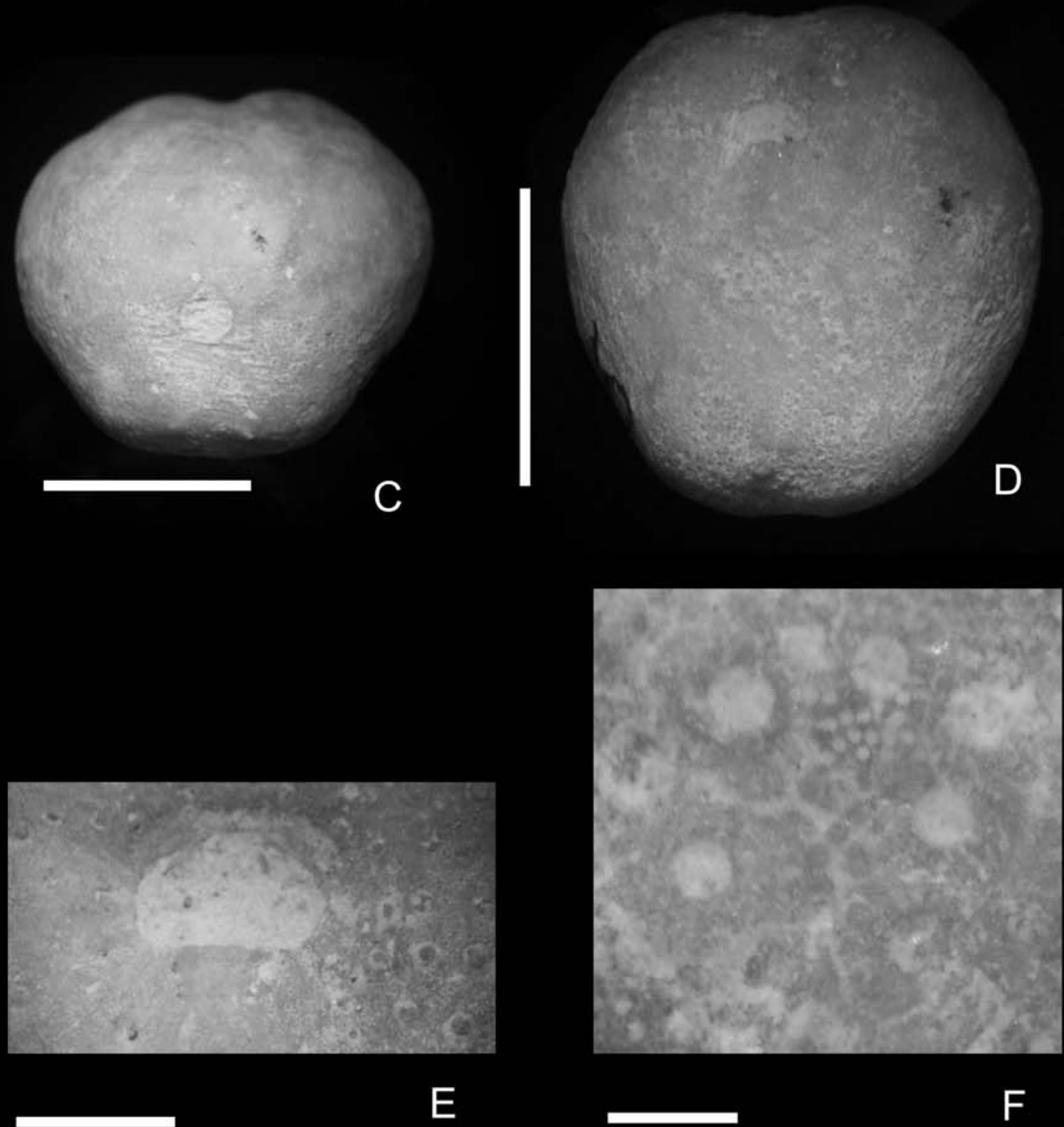

E

Figura 5 - Hemiaster proclivus Cotteau, Peron \& Gauthier do Albiano (Membro Romualdo, Formação Santana) da Bacia do Araripe, Pernambuco: exemplar FPH-1535-I.A - vista aboral; exemplar FPH-1532-I. B - vista lateral; $C$ - vista posterior; D - vista oral; $E$ - vista do peristômio; $F$ - vista do sistema apical. Barra de escalas: $A, B, C, D=10 \mathrm{~mm} ; E, F=1 \mathrm{~mm}$. 
Tabela 1 - Medidas (em milímetros) da carapaça de Hemiaster proclivus Cotteau, Peron \& Gauthier: $C=$ comprimento; $L=$ largura; $A=$ altura; $P A=$ comprimento das pétalas anteriores; $P P=$ comprimento das pétalas posteriores; $D A B A=$ distância do disco apical ao bordo anterior da carapaça.

\begin{tabular}{|c|c|c|c|c|c|c|c|c|c|}
\hline $\mathbf{N}^{0}$ & C & $\mathbf{L}$ & $L / C$ & A & $\mathrm{A} / \mathrm{C}$ & PA & PP & DABA & DABA/C \\
\hline 1 & 24,5 & 23,0 & 0,93 & 16,0 & 0,65 & 7,5 & 4,5 & 13,0 & 0,53 \\
\hline 2 & 24,0 & 22,5 & 0,93 & 18,5 & 0,75 & 7,0 & 4,0 & 13,0 & 0,54 \\
\hline 3 & 22,0 & 21,0 & 0,95 & 15,5 & 0,70 & 7,0 & 4,1 & 11,5 & 0,52 \\
\hline 4 & 19,5 & 18,2 & 0,93 & 13,5 & 0,69 & 6,0 & 3,5 & 10,0 & 0,51 \\
\hline 5 & 19,0 & 18,0 & 0,94 & 13,5 & 0,71 & 6,0 & 3,8 & 10,0 & 0,52 \\
\hline 6 & 18,0 & 17,5 & 0,97 & 12,5 & 0,69 & 5,2 & 3,5 & 9.0 & 0,50 \\
\hline 7 & 17,0 & 16,0 & 0,94 & 13,5 & 0,79 & 5,0 & 2,5 & 9,0 & 0,52 \\
\hline 8 & 15,5 & 14,5 & 0,93 & 11,5 & 0,74 & 4,9 & 3,0 & 8.0 & 0,51 \\
\hline 9 & 15,0 & 14,0 & 0,93 & 11,0 & 0,73 & 3,9 & 2,0 & 8.0 & 0,53 \\
\hline 10 & \multicolumn{9}{|c|}{ leve compressão lateral } \\
\hline 11 & 14,0 & 14,0 & 1,0 & compres & 1/aboral & 3,2 & 2,0 & 7,5 & 0,53 \\
\hline 12 & 17,5 & 16,0 & 0,91 & - & - & 5,0 & 3,0 & - & - \\
\hline
\end{tabular}

a oval, contornado por uma margem espessa. Os filódios são formados por isoporos separados por uma estrutura semelhante aquela que divide os isoporos do ambulacro III.

O plastrão é largo e triangular, com tubérculos maiores situados na região mediana e tubérculos menores em direção ao bordo posterior. A placa labral, observada no exemplar FPH-1527-I, é longa e triangular.

Distribuição estratigráfica e geográfica: de acordo com Smith em Smith \& Bengtson (1991), esta espécie foi descrita originalmente de estratos do Cenomaniano de Batna, Argélia, sendo também conhecida no sul da França. No Brasil, a espécie é conhecida do Eoalbiano ao Neoalbiano da Formação Riachuelo aflorante no Estado de Sergipe, Bacia de Sergipe-Alagoas (Smith \& Bengtson 1991, Manso 2003). No presente trabalho, Hemiaster proclivus Cotteau, Peron \& Gauthier têm sua distribuição geográfica ampliada, passando a ser registrada também no Albiano da Formação Santana (Membro Romualdo), na porção oeste da Bacia do Araripe, em Pernambuco.

Observações: Smith em Smith \& Bengtson (1991) observou, que nos exemplares de $H$. proclivus da bacia de Sergipe, o fascíolo peripetálico é parcialmente desenvolvido desaparecendo na região anterior da carapaça onde é substituído por tubérculos maiores. Em nossos exemplares foi observada a presença deste fascíolo também na região anterior da carapaça. De acordo com Néraudeau et al. (1998), a morfologia dos fascíolos é estabelecida muito cedo na ontogenia, e por volta dos $4 \mathrm{~mm}$ de comprimento, o modelo dos tubérculos que formam os fascíolos são bastante estáveis, apesar das mudanças na arquitetura e medidas da carapaça. Por vezes, durante o crescimento das carapaças, as bandas de fascíolos podem se reorganizar, expandindo-se para outras placas ou mesmo desaparecendo, considerando-se ainda que a largura da banda fasciolar aumenta durante toda a vida do ouriço-do-mar (Néraudeau et al. 1998). De acordo com estes autores, a distribuição de determinado tipo de fascíolo na carapaça pode variar num mesmo indivíduo. Esta variação foi observada, por exemplo, por Smith (Smith \& Bengtson 1991) em Hemiaster (Leymeriaster) leymeriei, onde há um orthofascíolo peripetálico de um lado das pétalas e um parafascíolo do outro. Os fascíolos dos equinoides também podem variar como uma adaptação às mudanças ambientais, em especial em resposta ao tamanho das partículas do substrato (Zaghbib-Turki 1990). Este autor observou diferenças na distribuição de fascíolos em espécies de Mecaster do Cenomaniano da Tunísia, onde formas que teriam vivido em ambiente com substrato formado por areia grossa mostravam parafascíolos, e espécimes que teriam vivido no interior de sedimentos finos apresentavam orthofascíolos. Neumann (1996) discutiu o caráter taxonômico dos fascíolos nas famílias Toxasteridae e Hemiasteridae do Eocretáceo, assinalando que a presença e morfologia desta estrutura são altamente variáveis, e concluindo que os fascíolos não devem ser utilizados como uma feição diagnóstica para a diferenciação de espécies.

CONSIDERAÇÕES PALEOECOLÓGICAS Os equinoides 'irregulares', desde a sua evolução a partir de formas 'regulares' (Kier 1972, 1974), têm explorado 
diferentes habitats no interior dos sedimentos. Para tanto, estas formas precisam ter dispositivos que facilitem sua respiração, alimentação e deposição de dejetos, que podem variar de acordo com o tamanho dos grãos do sedimento (Kanazawa 1992). Este autor observou que o formato das carapaças dos espatangoides, grupo ao qual pertencem às formas aqui descritas, pode possibilitá-los a viver em diferentes substratos, favorecendo seu enterramento ora mais próximo ora mais afastado da superfície sedimento-água.

Carapaças relativamente planas e baixas, de acordo com Smith (1984), sugerem uma adaptação a substratos mais estáveis ao movimento das águas. Douvillaster benguellensis apresenta uma carapaça bastante baixa e assim provavelmente teria tido um modo de vida epifaunal a semiendofaunal, como discutido por Manso \& Souza-Lima (2003). Kanazawa (1992) observou que espatangoides que possuem uma carapaça com a superfície ventral plana invariavelmente se enterram próximo à superfície do sedimento. Normalmente seus espinhos dorsais são utilizados para a defesa do animal e os latero-ventrais para auxiliar a voltarem à posição normal, quando são revirados pela ação de correntes ou ondas.

Como observado por Manso \& Souza-Lima (2003) em Douvillaster benguellensis em Sergipe, o pouco oxigênio dissolvido no meio e disponível à vida é o nítido controle faciológico observado nas ocorrências de $D$. benguellensis na Bacia de Sergipe-Alagoas, onde esta espécie ocorre exclusivamente nos folhelhos e sítios argilosos gerados em ambientes de baixa energia do Membro Taquari da Formação Riachuelo. O Atlântico durante o Aptiano-Albiano na região da Bacia de Sergipe-Alagoas parece ter sido um longo e estreito golfo com restrições à circulação marinha ampla e localizadas condições anóxico-disóxicas, estas devidas à grande variabilidade na topografia do fundo (Manso \& Souza-Lima 2003). D. benguellensis desenvolveu estruturas adaptadas a um ambiente pobre em oxigênio ampliando a superfície da área dos pódios e as aberturas dos poros, de forma a absorver o máximo de oxigênio possível. A sequência sedimentar do Membro Romualdo na Bacia do Araripe também representa a deposição em um golfo, enseada ou sistema de lagunas costeiras, com restrições de circulação onde $D$. benguellensis teria vivido durante o Albiano. Bruno \& Sial (2009) ao estudarem o biválvio Pseudoptera Meek 1873, ocorrente em camadas equivalentes da Formação Santana na porção leste da bacia, observaram indícios de um ambiente de vida marinho raso, calmo e bem oxigenado, com influxo de águas continentais.

Equinoides com carapaças que possuem a altura posterior maior do que a altura anterior possui maior capacidade de se enterrarem no sedimento, envolvendo mais movimentos repetidos para frente do que propriamente movimentos de escavação (Kanazawa 1992). Smith (1984) observou que esta diferença entre a altura posterior e anterior da carapaça facilita o seu enterramento principalmente em sedimentos de granulometria fina. Já Mcnamara \& Philip (1980) salientaram que quanto maior for a diferença entre as alturas anterior e posterior da carapaça, mais fino é o sedimento onde os equinoides vivem. Em Hemiaster proclivus, a diferença de altura entre a região anterior e a posterior é relativamente pequena, indicando que possivelmente teriam vivido em substratos compostos por areia de granulometria média. Outras feições morfológicas da carapaça de Hemiaster proclivus oferecem elementos para deduzir seu modo de vida. Com base nos trabalhos de Kanazawa (1992), Néraudeau et al. (1998), Smith $(1984,1995)$ e Manso (2003), os tubérculos de tamanhos diferentes sobre a carapaça indicam que seus espinhos dorsais, maiores na região anterior, provavelmente tinham a função de empurrar o animal para fora do sedimento. Os petaloides levemente sulcados parecem ter sido pouco eficientes para trocas gasosas, de modo que esta espécie deveria ter vivido junto à superfície, em sedimentos de granulação média. A presença de parafascíolo peripetálico também na extremidade distal do ambulacro anterior indica que estes pequenos espinhos protegiam esta região e as pétalas pares de elementos mais finos, promovendo uma leve corrente de água para facilitar as trocas gasosas. Os poros adapicais, semelhantes aos adambulacrais, não sugerem a presença de pódios construtores de funil. Assim, em $H$. proclivus, a água contida no interior do sedimento deve ter sido utilizada também para trocas gasosas. O pequeno periprocto indica que nesta espécie teria havido pequenas descargas fecais, que teriam sido depositadas fora do sedimento por um funil sanitário, cuja presença é evidenciada pelos poros abaixo do periprocto. O raso sulco ambulacral anterior e os pronunciados pares de filódios em torno do peristoma indicam que os pódios bucais teriam sido os principais responsáveis pelo processo de alimentação, coletando partículas do interior do sedimento.

Os estratos albianos da Formação Riachuelo da Bacia de Sergipe-Alagoas onde ocorre Hemiaster proclivus (Manso 2003) compreendem a deposição sob leques subaquosos costeiros (Membro Angico) com intercalações de calcilutitos e folhelhos depositados em áreas mais calmas e profundas (Membro Taquari) e calcarenitos, calciruditos oncolíticos e calcáreos oolíticos formados em recifes algálicos e patch reefs isolados, representando ambientes costeiros de alta energia (Membro Maruim). Na Bacia do Araripe, Hemiaster proclivus ocorre em sua porção oeste, em áreas próximas de onde foi relatada a presença de outras espécies de equinoides, tanto os cassiduloides Bothryopneustes araripensis 
(Beurlen 1966) e Pygurus tinocoi Beurlen 1966 como o espatangoide Douvillaster benguellensis, indicando que provavelmente dividiam o mesmo paleoambiente marinho restrito, mas ocupando diferentes nichos caracterizados por variações granulométricas e disponibilidade de oxigênio. Mesmo assim, os cassiduloides teriam participado da epifauna ou semiendofauna (Manso \& Hessel 2007), e os espatangoides participariam da endofauna: Hemiaster proclivus viveria em áreas mais rasas e Douvillaster benguellensis teria dominado áreas mais profundas, com menor circulação hídrica.

CONCLUSÕES O estudo de novos exemplares de equinodermos espatangoides coletados nos estratos mais superiores da Formação Santana (Membro Romualdo) aflorantes na Fazenda dos Isaques no município de Rancharia, Estado de Pernambuco, porção oeste da Bacia do Araripe, permite listar as seguintes principais conclusões:

a) a ocorrência da espécie Douvillaster benguellensis (Loriol 1888) nos estratos albianos da Bacia do Araripe em Pernambuco amplia sua distribuição geográfica para áreas mais setentrionais, considerando que até o momento era conhecida apenas nos estratos neoaptianos da Formação Riachuelo (Membro Taquari) da Bacia de Sergipe-Alagoas (Brasil) e neoalbianos na Formação Catumbela da Bacia de Benguela (Angola);

b) Douvillaster benguellensis apresenta uma carapaça bastante baixa com a superfície ventral plana, provavelmente tendo tido um modo de vida semiendofaunal, se enterrando bastante próximo à superfície em áreas mais profundas de um golfo ou enseada, com menor oxigenação e circulação hídricas;

c) a ocorrência mais abundante de representantes da espécie Hemiaster proclivus Cotteau, Peron \& Gauthier 1878 nas camadas albianas da Bacia do Araripe em Pernambuco amplia sua distribuição geográfica para oeste, pois antes havia sido registrada no Albiano da Bacia de Sergipe-Alagoas (Formação Riachuelo: membros Angico, Taquari e Maruim) e Cenomaniano da Argélia e do sul da França;

d) a diferença de altura entre as regiões anterior e posterior da carapaça de Hemiaster proclivus é relativamente pequena, indicando que esta espécie possivelmente vivia endofaunalmente próximo à superfície de substratos arenosos de granulometria média em áreas marinhas bastante rasas;

e) na porção oeste da Bacia do Araripe, os espatangoides Hemiaster proclivus e Douvillaster benguellensis ocorrem em áreas próximas de onde foi relatada a presença de outras espécies de cassiduloides (Bothryopneustes araripensis (Beurlen 1966) $e$ Pyguros tinocoi Beurlen 1966), indicando que provavelmente estes equinoides dividiam o mesmo paleoambiente marinho restrito, mas ocupando diferentes nichos caracterizados por variações granulométricas e de disponibilidade de oxigênio.

Agradecimentos Nossos agradecimentos a Wagner Souza Lima (Fundação Paleontológica Phoenix) pelas proveitosas discussões e revisão do inglês, e a Ana Paula Bruno e Sonia Maria Agostinho (UFPe) pela companhia no trabalho de campo em Pernambuco.

\section{Referências}

Arai M., Carvalho I.S., Cassab R.C.T. 2004. Bacia do Araripe. Phoenix, 6(72):1-6.

Arai M. \& Coimbra J.C. 1990. Análise paleoecológica do registro das primeiras ingressões marinhas na Formação Santana (Cretáceo Inferior da chapada do Araripe). In: DNPM/SBP/SBG, Simpósio Bacia do Araripe e Bacias Interiores do Nordeste, 1, Atas, p. 225-239.

Assine M.L. 1992. Análise estratigráfica da Bacia do Araripe, Nordeste do Brasil. Rev. Bras. Geoc., 22(3):289-300.

Assine M.L. 2007. Bacia do Araripe. Boletim de Geociências da Petrobras, 15(2):371-389.

Bengtson P. 1983.The Cenomanian-Coniacian of the Sergipe Basin, Brazil. Fossils and Strata, 12:1-78.

Beurlen K. 1966. Novos equinóides do Cretáceo do Nordeste do Brasil. Anais da Academia Brasileira de Ciências, 38(3-4):455-464.

Brito I.A.M. 1981. O gênero Hemiaster no Cretáceo do Brasil (Echinoidea, Spatangoidea). In: Congr. LatinoAmericano de Paleontologia, 2, Anais, p. 399-414.

Brito I.A.M. 1990. O Cretáceo e sua importância na geologia do Brasil. Tese de Doutorado, Instituto de Geociências, Universidade Federal do Rio de Janeiro, Rio de Janeiro, 95 p.

Bruno A.P. \& Sial A.N. 2009. Ambiente de vida de Pseudoptera (Bivalvia, Bakelliidae) da Formação Romualdo, Bacia do Araripe. Boletim da Sociedade Brasileira de Paleontologia, 24(62):13.

Chagas D.B. 2006. Litoestratigrafia da Bacia do Araripe: reavaliação e propostas para revisão. Dissertação de Mestrado, Instituto de Geociências e Ciências Exatas, Universidade Estadual Paulista, Rio Claro, 127 p.

Coimbra J.C., Arai M., Carreño A.L. 2002. Biostratigraphy of Lower Cretaceous microfossils from the Araripe Basin, northeastern Brazil. Geobios, 35(6):687-698.

Cotteau G., Peron P., Gauthier V. 1878. Echinides fossiles de l'Algerie: description des especes deja recueillies dans ce pays et considerations sur leur position stratigraphique. 4, Etage Cenomanien (premiere partie). Paris, G. Masson, 245 p.

Heimhofer U., Ariztegui D., Lenniger M., Hesselbo S.P., 
Martill D.M. 2010. Deciphering the depositional environment of the laminated Crato fossil beds (Early Cretaceous, Araripe Basin, Northeastern Brazil). Sedimentology, 57:677-694.

Kanazawa K. 1992. Adaptation of test shape for burrowing and locomotion in spatangoid echinoids. Palaentology, 35(4):733-750.

Kier P.M. 1972. Tertiary and Mesozoic Echinoids of Saudi Arabia. Smithsonian Contrib. Paleobiol., 10: 1-105.

Kier P.M. 1974. Evolutionary trends and their functional significance in the post-Paleozoic echinoids. Jourl Paleont., 48(3):1-95.

Lambert J. 1931. Étude sur les échinides fossiles du Nord de l’Áfrique. Mém. Soc. Géol. France, 7(2):1-108.

Lambert J. 1932. Étude sur les échinides fossiles du Nord de l’Áfrique. Mém. Soc. Géol. France, 7(4):109-228.

Loriol P. 1888. Materiaux pour l'etude stratigraphique et paléontologique de la Province d'Angola: description des Échinides. Mém. Soc. Phys.Hist. Nat. Genève, 30(2):97-114.

Manso C.L.C. 2003. Paleoecologia, paleobiogeografia e sistemática dos equinóides do Aptiano-Albiano (Cretáceo) do Brasil. Tese de Doutorado, Instituto de Geociências, Universidade Federal da Bahia, Salvador, 206 p.

Manso C.L.C. \& Hessel M.H. 2007. Revisão de Pygidiolampas araripensis (Beurlen, 1966), (Echinodermata Cassiduloida) da Bacia do Araripe, nordeste do Brasil. Geociências, 26(3):271-277.

Manso, C.L.C. \& Souza-Lima, W. 2003. O equinóide Douvillaster Lambert 1917 na Formação Riachuelo, Sub-bacia de Sergipe, Brasil. Rev. Bras.Paleo., 5:29-37.

Martill D.M., Bechly G., Loveridge R.F. 2007. The Crato fossil beds of Brazil: Window into an ancient world. PHD Tesis, Cambridge University, Cambridge, 625 p.

Maury C.J. 1937. O Cretáceo de Sergipe. Rio de Janeiro, SGMB, 283 p.

Mcnamara K.J. \& Philip G.M. 1980. Living Australian schizasterid echinoids. Proc. Linn. Soc. New South
Wales, 104(2):127-146.

Néraudeau D., David B., Madon C. 1998. Tuberculation in spatangoid fascioles: Delineating plausible homologies. Lethaia, 31:323-334.

Neumann C. 1996. The mode of life and paleobiogeography of the genus Douvillaster Lambert (Echinoidea: Spatangoida) as first recorded in the Lower Cretaceous (Albian) of Spain. Berl. Geowiss. Abh., E18:257-265.

Santos M.E.C.M. \& Cunha F.L.S. 1959. Sobre Hemiaster jacksoni Maury e outros equinóides do Cretáceo brasileiro. Rio de Janeiro, DNPM/DGM, Boletim 186, 19 p.

Silva M.A.M. 1986. Lower Cretaceous unconformity truncating evaporite-carbonate sequence, Araripe Basin, northeastern Brazil. Rev. Bras. Geoc., 16(3):306-310.

Smith A.B. 1984. Echinoid palaeobiology. London, George Allen \& Unwin, 199 p.

Smith A.B. 1991. Taxonomic descriptions. In: Smith, A.B. \& Bengtson, P. 1991. Cretaceous echinoids from northeastern Brazil. Fossils and Strata, 31:16-64.

Smith A.B. 1995. Late Campanian-Maastrichtian echinoids from the United Arab Emirates-Oman border region. Bull.Nat. Hist.Mus., 51(2):121-240.

Smith A.B. 2011. The Echinoid Directory. Disponível em: http://www.nhm.ac.uk/palaeontology/echinoids. Acessado em: 01/07/2011.

Smith A.B. \& Bengtson P. 1991. Cretaceous echinoids from north-eastern Brazil. Fossils and Strata, 31:1-88.

White C.A. 1887. Contribuições à Paleontologia do Brasil. Arch. Mus. Nac. RJ, 7:1-273.

Zaghbib-Turki D. 1990. Stratégie adaptative des Hemiaster du Crétace Supérieur (Cénomanien-Coniacien) de la plateforme carbonatée de Tunisie. In: DeRidder C., Dubois P., Lahaye M.C.Y., Jangoux M. (eds) Echinoderm Research. Rotterdam, Balkema, p. 49-56.

Manuscrito ID 21634 Submetido em 05 de junho de 2011 Aceito em 07 de fevereiro de 2012 